\title{
Effectiveness of the Instructional Module on Knowledge and Interpretation of ABGs Among Critical Care Nurses
}

\author{
Umar Zeb*, Said Alam, Farman Ali, Mohammad Hanif, Sardar Ali \\ Institute of Nursing Sciences, Khyber Medical University Peshawar, Khyber Pakhtunkwa, Pakistan \\ Email address: \\ rose.baina@gmail.com (U.ZZeb), alam.kmu@gmail.com (S.Alam), farman.bakar@gmail.com (F. Ali),md_hanif7@yahoo.com (M. Hanif), \\ sardarqayum@gmail.com (S. Ali) \\ *Corresponding author
}

\section{To cite this article:}

Umar Zeb, Said Alam, Farman Ali, Mohammad Hanif, Sardar Ali. Effectiveness of the Instructional Module on Knowledge and Interpretation of ABGs Among Critical Care Nurses. American Journal of Nursing and Health Sciences. Vol. 10, No. 5, 2021 , pp. $216-221$. doi: 10.11648/j.ajns.20211005.11

Received: January 15, 2021; Accepted: May 27, 2021; Published: September 26, 2021

\begin{abstract}
Arterial Blood Gases interpretation are considered as one of the significant investigations in the critical care areas that guides in appropriate direction to make better decision for diagnosis, treatment and expected outcome of the concerned disorders. Nurses are considered as the primary care providers in critical care units therefore their knowledge regarding ABGs interpretation and analysis may play a vital role in the management of the seriously ill patient admitted with respiratory, cardiovascular, acid base disorder and renal disease. The main intention of the study was to appraise the Effectiveness of the Instructional Module on Knowledge and Interpretation of ABGs among Critical Care Nurses. A quantitative quasiexperimental study design was used with a sample of $30 \mathrm{CCN}$ by census method from Ayub Teaching hospital Abbottabad. Data was collected through a reliable and validated questionnaire before and after assigning with the instructional module. Data was analyzed by SPSS-22. The study result showed that the pretest knowledge score of the critical care nurses in the pre stage intervention was $53.3 \%$ which was poor on the grading scale. Overall mean score was found as $51.9963+26.54$ in the pre assessment phase while $74.6333+15.98$ was reported in the post phase of giving the instructional module with a mean significant difference of $22.63+39.50$. Statistically the instructional module was found effective in enhancing the nurse's knowledge with a -value of 0.004 . The findings of the study depict that instructional module has a positive and significant association with the level knowledge of nurses regarding ABGs interpretation.
\end{abstract}

Keywords: Arterial Blood Gases, Critical Care Nurses, Interpretation, Knowledge

\section{Introduction}

Respiratory, cardiovascular, metabolic and kidneys diseases have prevailed drastically in recent years and they are becoming the global concerns of admission to the hospitals. Morbidity and mortality rates of respiratory, cardiovascular and kidneys problems are increasing day by day. Majority $55 \%$ patients in the critical care area are suffering from respiratory failure. [1] Approximately 137 patients are hospitalized per 100000 population per year due to respiratory failure in an advanced country like United States of America. [2] Chronic kidney diseases affect $10 \%$ of the population around the world and a great number of people die annually. [3] According to the cardiovascular fact sheet, around 17.7 million deaths occurred in the year 2015 which showed around $31 \%$ of all the deaths occurred worldwide. $[1,2]$

Various parameters and diagnostic tests are carried out to monitor the respiratory, cardiovascular and kidney status of such patients. Arterial Blood Gases are considered as one of the most used methods in critical care units by the health professionals to observe the patients for respiratory, nephrology and cardiovascular complications in terms of looking into oxygenation and ventilation of body, respiratory and metabolic acidosis and alkalosis. [4] Furthermore, it also gives us information about serum electrolyte potassium and sodium level. [5] ABGs are considered as one of the key investigations in the critical care unit which give us direction for proper diagnosis, treatment, response of the patient 
towards treatment and expected outcomes. [6] It has been found that 8 of every 10 patients have an acid-base disorder in the critical areas and if not managed and addressed effectively by the nurses it would certainly lead to health miseries and sufferings. If there would be a small error in these interpretations it may lead to the life-threatening medical decision. [7]

Therefore, knowledge of Arterial Blood Gases interpretation is essential skill for the nurses where they must have to be knowledgeable which help them in the provision of best possible care to the patients in the critical care units. As Critical care units are the most challenging areas of the hospitals for the nurses because patients in life-threatening conditions are treated by them with specialized skills and nursing care. They are considered as the primary care providers in critical care units in the management of the seriously ill patient. [8] Although nurses play a vital role in the constant observation; performing skills and provision of best possible care in critical care units however nurses have been found poor in their knowledge regarding ABGs interpretation in various studies therefore, they need to be updated with proper instructional and practical training for the provision of best possible care to the patients admitted in critical care units with respiratory or metabolic disorders.

No available published study has been found in Pakistan regarding nurse's knowledge assessment so far to address this imperative issue. Therefore, this study may provide the baseline information and will help to identify the needs of nurses to assess and address the gaps in knowledge regarding ABGs interpretation.

Research objective for this study was, to evaluate the effectiveness of the instructional module on knowledge and interpretation of arterial blood gases among critical care nurses.

Research Question: what is the effect of the instructional module on knowledge and interpretation of arterial blood gases among critical care nurses?

Research Hypothesis:

$\mathrm{H}_{0}$ : There is no effect of the instructional module on knowledge and interpretation of arterial blood gases among critical care nurses.

$\mathrm{H}_{1}$ : There is an effect of the instructional module on knowledge and interpretation of arterial blood gases among critical care nurses.

\section{Materials and Methods}

A Quantitative quasi-experimental pretest and posttest research design was used to complete the study. A total 30 critical care nurses were selected by census sampling method from Ayub teaching hospital Abbottabad. Data was collected by self-administered and validated questionnaire with Cronbach alpha with reliability (Mean Score=0.75) and Validity (Mean Value=0.92) Validity is the degree of the instrument to measure what it is assumed to be measure. [9] and Reliability means accurateness of the measurement which is mainly focused on constancy and consistency. [10, 11] A pilot study was done on $10 \%$ of the study sample. All registered critical care nurses were taken who have at least six-month experience in critical care units and willing to participate. Nurses, who were not actively involved in patients care like Managers, administrators etc. Nurses who were not willing to participate was excluded. The Questionnaire have demographic information and $2^{\text {nd }}$ section was consisting of Knowledge assessments tool for ABG interpretation. Three steps were followed to carry out the intervention.

Step-1: Questionnaire was distributed among nurses for pretest phase to collect the data.

Step-2: Instructional module was given to the study participants and a 60 minutes teaching session was conducted on the same module by the ICU expert staff. The content of teaching was all the basic information regarding arterial blood gases (ABGs).

Step-3: This was the final step to distribute the questionnaire for posttest after 2 weeks of the instructional module and teaching session on ABGs interpretation.

The collected data was analyzed by Software SPSS Version-22 for its proper presentation. The questionnaire was consisted of 30 items. A rating scale for this questionnaire was (Excellent Knowledge: Greater than $80 \%$ Good Knowledge: 65 - 80\% Average Knowledge: 60 - 64.9\% Poor Knowledge: Less than 50\%). [12] After analyzing data, the findings and results were distributed and plotted on graphs, charts and tables in the form of percentages and frequencies. Mean and standard deviation was calculated for continuous variables (Age). Percentages and Frequencies was calculated for (Gender, Qualification, and Working Unit) to make it concise, clear and convenient for understating. Statistical Tests (Chi square test) was applied to interpret association of categorical variables with the mean scores of the participant knowledge and paired $\mathrm{T}$ test was applied to compare the pretest posttest mean scores of the participant, knowledge.

\section{Result}

Presentation of Demographic Variable: Presentations of the demographic variables have been given below in details.

Table 1. Presentations of the demographic variables.

\begin{tabular}{|c|c|c|c|}
\hline Demographic Variables & Category & f & $\%$ \\
\hline \multirow{2}{*}{ Gender } & Male & 08 & 26.66 \\
\hline & Female & 22 & 73.3 \\
\hline \multirow[t]{2}{*}{ Age in Years } & $31 \pm 10.51($ Mean w & & \\
\hline & Diploma & 20 & 66.66 \\
\hline \multirow[t]{2}{*}{ Qualification } & Bachelor's Degree & 08 & 26.66 \\
\hline & Master's Degree & 02 & 6.66 \\
\hline
\end{tabular}




\begin{tabular}{llll}
\hline Demographic Variables & Category & f & \% \\
\hline & 01 to 5 Years & 14 & 46.66 \\
& 06 to 10 Years & 08 & 04 \\
Total Years of Experience & 11 to 15 Years & 04 & 16.66 \\
& 16 Years or above & 05 & 13.33 \\
Workshop on ABGs & Yes & 25 & 16.66 \\
& No & 17 & 83.33 \\
Experience in Critical Units & 01 to 5 Years & 11 & 56.66 \\
& 06 to 10 Years & 0 & 36.66 \\
& 11 to 15 Years & 3 & 0 \\
Working Unit & 16 Years \& above & 10 & 10.33 \\
& Medical ICU & 5 & 33.33 \\
& Surgical ICU & 4 & 16.66 \\
Specialty & Cardiac ICU & 9 & 13.33 \\
& Neuro ICU & 2 & 30.33 \\
& Others & 20 & 06.66 \\
& Midwifery & 5 & 66.66 \\
\end{tabular}

Analysis of The Knowledge Evaluation Section: This section would mainly focus on the pre and posttest knowledge scores of critical care nurses based on the overall score with frequencies and percentages of the validated questionnaire as follow:

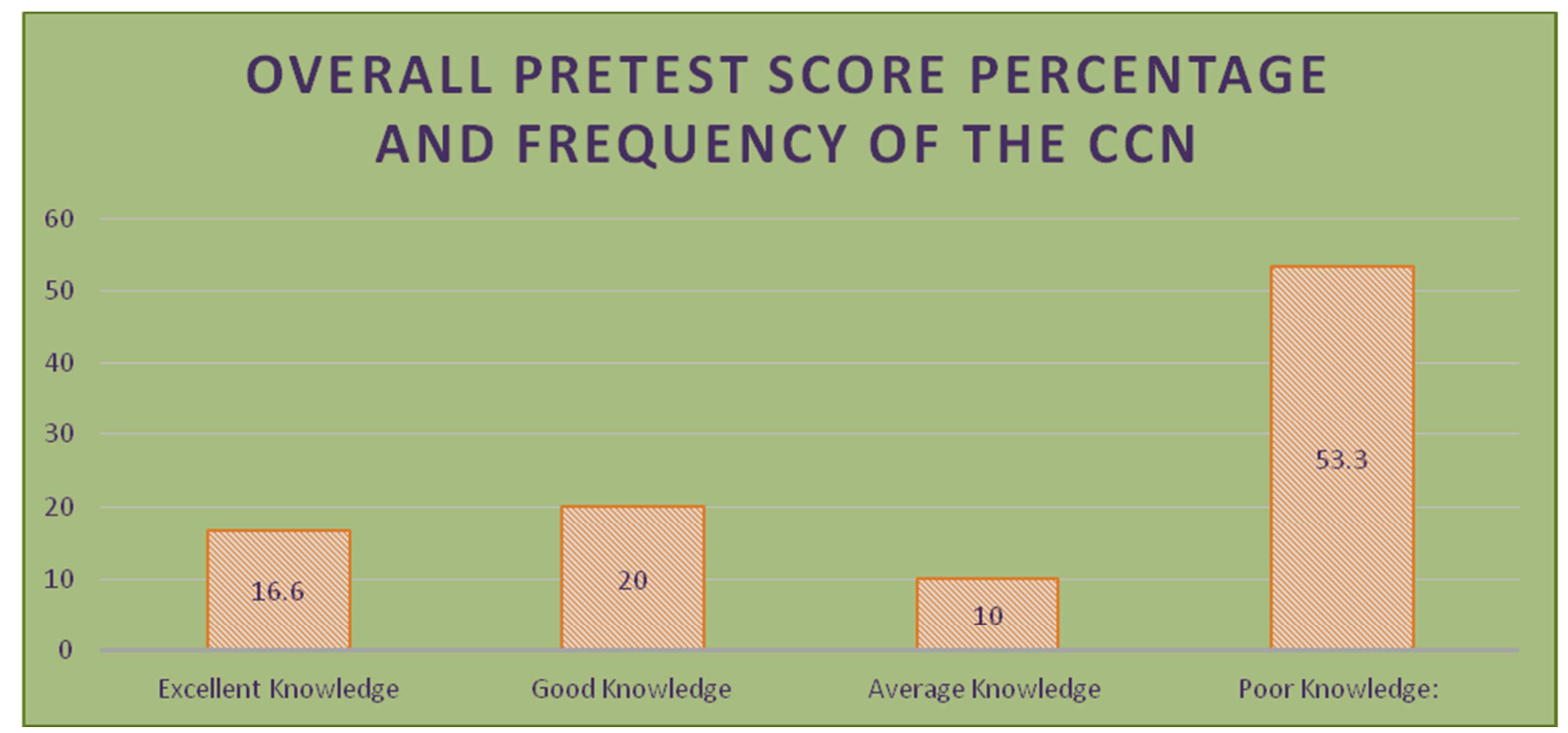

Figure 1. Pretest knowledge score of the critical care nurses.

The graph shows the frequency and percentage distribution of pretest knowledge score of the critical care nurses. The analysis showed that $16.66 \% \mathrm{CCN}$ had excellent knowledge and $20 \% \mathrm{CCN}$ had good knowledge. A very less number $(10 \%)$ of the $\mathrm{CCN}$ had average knowledge and majority
$(53.33 \%)$ of them had poor knowledge score in the pre phase of the intervention.

Association of Demographics \& Pre-Knowledge Score: Association was checked by Fisher Exact Test because of small sample size the result is shown in table below.

Table 2. Association of Demographics \& Pre-Knowledge Score.

\begin{tabular}{llll}
\hline No & Variables name & Fisher Exact Test-Value & P-Value \\
\hline 1 & Gender & 3.375 & 0.323 \\
2 & Qualification & 6.157 & 0.361 \\
3 & Total Years of Experience & 8.625 & 0.439 \\
4 & ABGs Workshop & 1.697 & 0.805 \\
5 & Total CCA Experience & 2.929 & 0.952 \\
6 & Working Unit & 13.307 & 0.196 \\
7 & Specialty & 10.142 & 0.191 \\
\hline
\end{tabular}

It was found that there were variations in the scores to certain extent by applying the statistical test as shown in the table. However, the p-value shows that those variations were not significant statistically. 


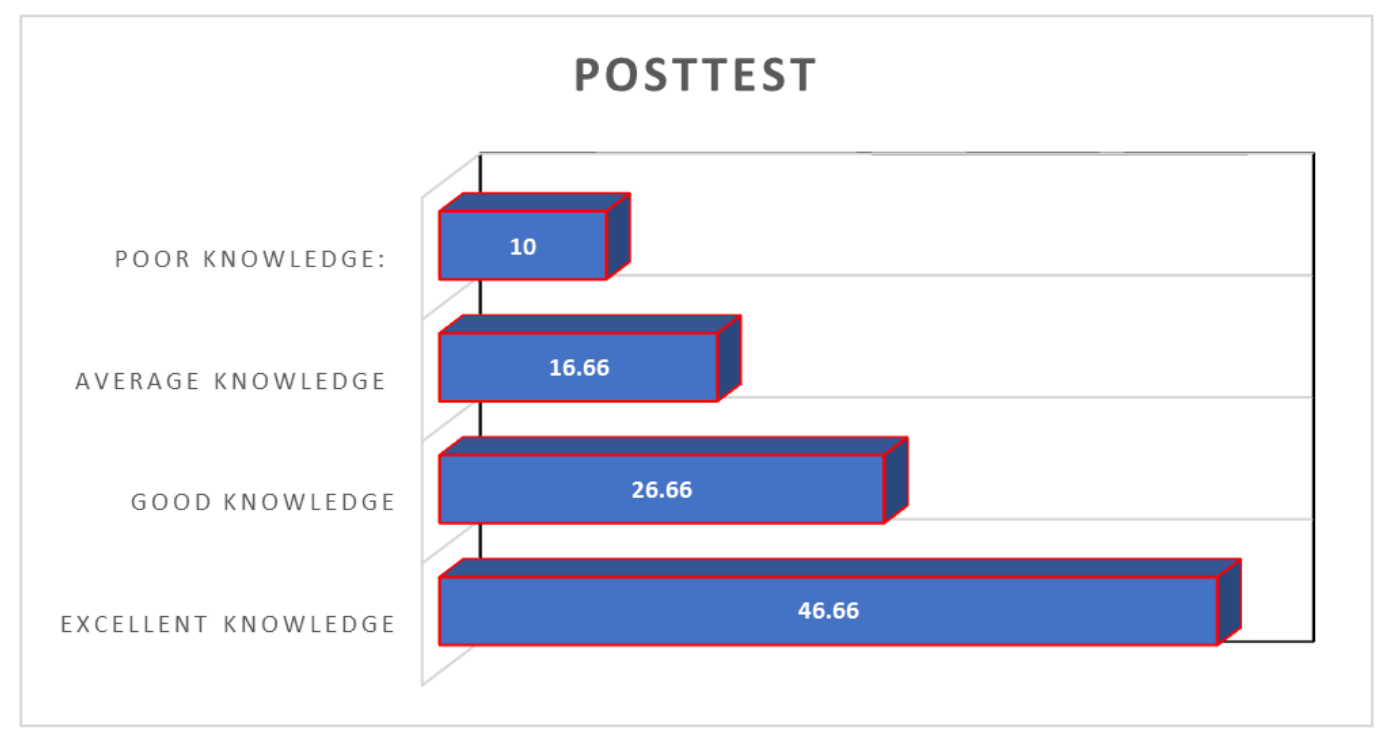

Figure 2. Overall Posttest Score Distribution of Knowledge.

Table 3. Association of variables with Posttest Knowledge.

\begin{tabular}{|c|c|c|c|c|}
\hline No & Variables Name & Fisher Exact Test Value & P-Value & Interpretation \\
\hline 1 & Gender & 1.261 & 0.928 & NS \\
\hline 3 & Qualification & 5.176 & 0.542 & NS \\
\hline 4 & Total years of Experience & 6.103 & 0.818 & NS \\
\hline 5 & ABGs Workshop & 1.521 & 0.808 & NS \\
\hline 6 & Total CCA Experience & 13.908 & 0.006 & Significant \\
\hline 7 & Working Unit & 6.390 & 0.982 & NS \\
\hline 8 & Specialty & 7.886 & 0.496 & NS \\
\hline
\end{tabular}

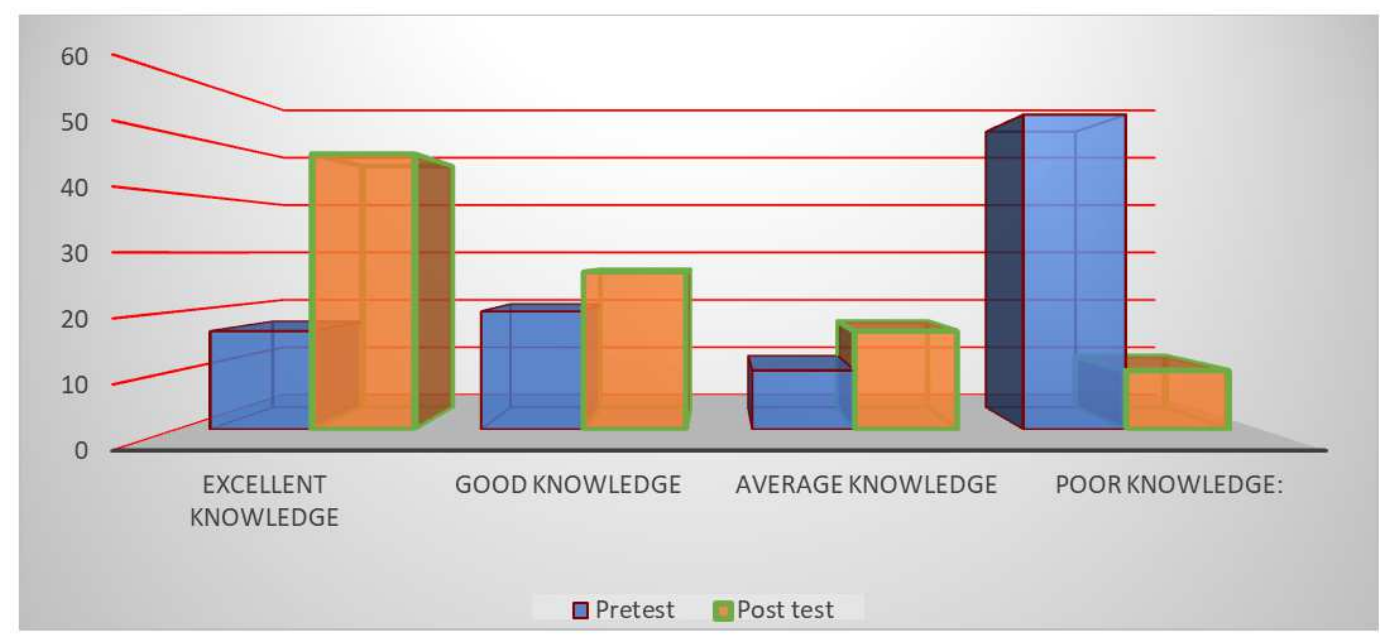

Figure 3. Graphical Presentation of the Comparison of Pretest and Posttest Knowledge.

Table 4. Comparison (Pretest \& Posttest Knowledge by Paired T-Test).

\begin{tabular}{|c|c|c|c|c|c|c|c|c|}
\hline \multicolumn{9}{|c|}{ PAIRED SAMPLES TEST } \\
\hline & \multicolumn{5}{|c|}{ PAIRED DIFFERENCES } & \multirow{3}{*}{$\mathbf{T}$} & \multirow{3}{*}{ Df } & \multirow{3}{*}{$\begin{array}{l}\text { Sig. (2 } \\
\text { tailed) }\end{array}$} \\
\hline & \multirow{2}{*}{ Mean } & \multirow{2}{*}{ Std. Deviation } & \multirow{2}{*}{ Std. Error Mean } & \multicolumn{2}{|c|}{ 95\% Confidence Interval of the Difference } & & & \\
\hline & & & & Lower & Upper & & & \\
\hline Pretest \& Post-test & 22.6370 & 39.50169 & 7.21199 & 37.38718 & -7.88682 & 3.139 & 29 & .004 \\
\hline
\end{tabular}

For more specific comparison paired t test was applied to get the inferential statistics about both the pre and post phases of the intervention. After the analysis a statistically significant mean difference of (22.63) was found in the means scores with a $p$ value of 0.004 . Based on current study findings the alternative hypothesis was accepted and the null hypothesis was rejected. 


\section{Discussion}

\subsection{Demographic Variables Discussion}

Demographic variables reported that most of the nurses $(73.33 \%)$ were female nurses in the current study while the mean age of the $\mathrm{CCN}$ was calculated as 31 years. Majority of nurses $46 \%$ had up to 5 years of clinical experience. Based on qualification majority $(66 \%)$ of the study participants had diploma in nursing. The current study findings were somehow parallel to the study conducted by Kaur and Charan [15]. According to the findings they reported majority (90\%) of female nurses while $93.3 \%$ of the participants had age up to 30 years of age in their study. $60 \%$ of the nurses had experience less than or up to 5 years of their clinical experience. However, the educational level was not found congruent to the current study findings as majority (88\%) of the participants of the study had diploma in general nursing according to their study. [13] The finding of this study has also been found parallel to another study conducted by Youssef (2013) reports the same statistics of demographics. According to the findings $85 \%$ of the participants were female nurses in their study with a mean age of 30 years and with majority of diploma qualification. Furthermore, regarding experience two-third of the nurses had 1 to 10 years of experience and $55 \%$ of them were working in the medical intensive care unit. And in contrast to the current study results none of the participants had got any training regarding ABGs interpretation. [14] Regarding workshop and training on $\mathrm{ABGs}$ interpretation $83 \%$ of the participants were found in the current study that they had not attended any such type of programmed only $17 \%$ have attended.

These findings were very consistent to the study conducted by Safwat (2018) where it was reported that $80 \%$ of the study participants had no exposure attend any course, workshop or training regarding the ABGs interpretation. [15]

\subsection{Comparison of The Pretest and Posttest Knowledge Score}

Pretest and posttest mean were compared. The mean pretest knowledge score was 51.9963 and mean knowledge score of posttests was 74.6 the difference between the pretest and posttest was 22.6 and the calculated $t$ value was 3.13 which was statistically significant at p-value 0.000 . Based on the current study finding the alternative hypothesis was accepted and the null hypothesis was rejected. According to the assessment of the critical care units' nurses in the pretest knowledge phase it was found that there were few participants in the excellent category of knowledge. Only a small fraction (16\%) of the nurses scored excellent knowledge while majority of them $(53.3 \%)$ scored poor knowledge according the assessment on the 30 items validated questionnaire. Further the overall mean pretest knowledge score was found as $(51.9963 \%)$ among the critical care nurses regarding the analysis and interpretation of the Arterial Blood Gases. Similarly, in the Post Test Phase it was found that significantly the results were changed from $16 \%$ to $46 \%$ excellent category while $53.3 \%$ of the poor knowledge category dropped to only $10 \%$. The same way the other categories of good knowledge and average knowledge were changed. Further after the intervention of study module and teaching session the overall mean score knowledge was improved with a mean value of $74.633 \%$ with a difference $22.6 \%$. Statistically at the level of significance of less than $0.05 \%$ the p-value was reported as $(0.000)$ that showed that nurses improved their level of interpretation significantly after handing over them the teaching material and conducting the session on ABGs analysis and interpretation.

Analyzing and evaluating the study findings in connection to the previous studies it was found that a study conducted by (Kaur, Charan 2018) and (Safwat 2018) shows the same patterns of pre and posttest knowledge. [13, 15] Another study conducted by Ganguly (2007) on ABGs analysis among nurses working in the critical care units found that the knowledge level of the nurses was unsatisfactory regarding ABGs interpretation. [16] The findings were also consistent with the study conducted by Schneiderman J. et al. (2009) to assess the efficiency of an online ABGs analysis computerbased learning module. The result showed that the nurse's knowledge was increased significantly while teaching them with an online learning programme. [17]

\subsection{Association of Demographics To Pre \& Posttest Knowledge}

Association of the pretest and posttest knowledge score was checked by fisher exact test The result showed that there was no significant association between the knowledge score of $\mathrm{CCN}$ in pretest and posttest except there was association showed in posttest knowledge score with total experience at the critical area where the $\mathrm{p}$ values calculated was obtained 0.006 which was less than $p$ tabulated value 0.05 . The parallel results were found in the study of Kaur and Charan (2018) where the demographic variables had no strong and statistically significant association with the pre and posttest knowledge. However, in contrast to the current study finding the study conducted by Safwat (2018) showed that demographic variables including gender, level of education, experience and training had a positive and statistically significant correlation with the pre and post mean score knowledge score except age. The difference may be attributed to the educational background, practices of nurses, sample size, research setting, and hospital patients flow in their origin.

\section{Strengths and Limitation of the Study}

The study is helpful in guiding policy makers to arrange training sessions for nurses with different topics study. The study is conducted in one hospital only, it would have better generalization if have conducted in other hospitals.

\section{Recommendations}

In service education must be given to the nurses to update their knowledge, skills and practices regarding ABGs 
interpretation and diagnosis related ABGs parameters. Seminars should be arranged frequently to highlight the importance of ABGs interpretation in the problem's identification of the patients. Study modules, written protocols and Workshops must be planned to bring quality into the hands on and practical skills of nurses towards better analysis of the arterial blood gases. Latest knowledge, skills and technology may be incorporated to keep the nurses with the current trends of medical practices in critical care units.

\section{Conclusion}

The findings of the study concluded that nurses were not up to the mark in order to analyze and interpret the ABGs as per the required standards and protocols. Therefore, continuous training in terms of giving instructions, written materials in the form of study modules and teaching sessions may have to plan frequently to ensure better care services in the critical care units.

\section{References}

[1] Bartal M. Health effects of tobacco use and exposure. Monaldi archives for chest disease. 2001; 56 (6): 545-54.

[2] Urden LD, Stacy KM, Lough ME. Critical Care Nursing-E-Book: Diagnosis and Management: Elsevier Health Sciences; 2017.

[3] Mannino D, Ford E, Redd S. Obstructive and restrictive lung disease and functional limitation: data from the Third National Health and Nutrition Examination. Journal of internal medicine. 2003; 254 (6): 540-7.

[4] Roger VL, Go AS, Lloyd-Jones DM, Benjamin EJ, Berry JD, Borden WB, et al. Heart disease and stroke statistics--2012 update: a report from the American Heart Association. Circulation. 2012; 125 (1): e2-e220.

[5] Coyer FM, Wheeler MK, Wetzig SM, Couchman BA. Nursing care of the mechanically ventilated patient: What does the evidence say?: Part two. Intensive and critical care nursing. 2007; 23 (2): 71-80.
[6] Asif M, Sarkar PK. Three-digit Allen's test. The Annals of thoracic surgery. 2007; 84 (2): 686-7.

[7] Mohammed HM, Abdelatief DA. Easy blood gas analysis: Implications for nursing. Egyptian Journal of Chest Diseases and Tuberculosis. 2016; 65 (1): 369-76.

[8] Lundberg GD. The need for an outcomes research agenda for clinical laboratory testing. JAMA. 1998; 280 (6): 565-6.

[9] Gerrish K, Lacey A. The research process in nursing: John Wiley \& Sons; 2010.

[10] Long T, Johnson M. Rigour, reliability and validity in qualitative research. Clinical effectiveness in nursing. 2000; 4 (1): 30-7.

[11] Researches. Nigerian Postgraduate Medical Journal. 2015; 22 (4): 195.

[12] Ali Sas, Taverner BC, Ghani M, Kussor Z, Naz S. Knowledge of triage among nurses in emergency units. Biomedica. 2013 29 (4): 240-3.

[13] Kaur A, Charan GS. A Study to Assess the Effectiveness of STP on Knowledge and Practice Regarding ABGs among ICU Nurses in Selected Hospitals at Jalandhar, Punjab.

[14] Youssef W, Yahia A, Ali NS, Elhabashy S. Factors Affecting Validity of Arterial Blood Gases Results among Critically Ill Patients: Nursing Perspectives. Journal of Education and practice. $2013 ; 4$.

[15] Safwat AM. Effectiveness of a computer-based learning module on arterial blood gas interpretation among staff nurses in critical care units. Innovative Journal of Medical and Health Science. 2018; 8 (3): 31-40.

[16] Ganguly S. Effectiveness of a need based teaching protocol on "Nurses Responsibility in ABG analysis" for the nursing personnel working in the critical care units. Asian Journal of Cardiovascular Nursing. 2007; 15 (2): 12-7.

[17] Schneiderman J, Corbridge S, Zerwic JJ. Demonstrating the effectiveness of an online, computer-based learning module for arterial blood gas analysis. Clinical Nurse Specialist. 2009; 23 (3): $151-5$. 